\title{
The effect of YAP expression in tumor cells and tumor stroma on the prognosis of patients with squamous cell carcinoma of the oral cavity floor and oral surface of the tongue
}

\author{
JOLANTA SZELACHOWSKA ${ }^{1,2}$, PIOTR DONIZY ${ }^{3}$, KATARZYNA RATAJCZAK-WIELGOMAS ${ }^{4}$, \\ AGNIESZKA HALON $^{3}$, DOMINIKA ZIELECKA-DEBSKA ${ }^{2,5}$, KRYSTIAN LICHON ${ }^{1,2}$, ADAM MACIEJCZYK ${ }^{2,6}$, \\ EWELINA LATA-WOZNIAK ${ }^{1,2}$, ALEKSANDRA PIOTROWSKA ${ }^{4}$ and RAFAL MATKOWSKI ${ }^{2,5}$ \\ ${ }^{1}$ Department of Oncology, Gynaecological Oncology Clinic, Wroclaw Medical University; ${ }^{2}$ Department of Radiotherapy, \\ Lower Silesian Oncology Centre, 53-413 Wroclaw; ${ }^{3}$ Department of Pathomorphology and Oncological Cytology, \\ Wroclaw Medical University, 50-556 Wroclaw; ${ }^{4}$ Histology and Embryology Division, Wroclaw Medical University, \\ 50-368 Wroclaw; ${ }^{5}$ Department of Oncology, Clinic of Surgical Oncology, Wroclaw Medical University, 53-413 Wroclaw; \\ ${ }^{6}$ Department of Oncology, Clinic of Radiation Oncology, Wroclaw Medical University, 53-413 Wroclaw, Poland
}

Received January 21, 2019; Accepted July 4, 2019

DOI: $10.3892 / 01.2019 .10695$

\begin{abstract}
Classic prognostic factors, such as clinical advancement of the disease and histological grade of the tumor, continue to have a decisive role in the selection of therapeutic strategy in patients with carcinoma of the oral cavity floor and oral surface of the tongue (OCC). YAP1/Yes-associated protein 1 (YAP) and transcriptional co-activator with PDZ-binding motif, WWTR1 (TAZ) proteins, appear to be promising markers that may be used to develop personalized therapies. The aim of the present study was to analyze the associations between the levels of YAP, TAZ and tyrosine-protein phosphatase non-receptor type 14 (PTPN14) and to determine whether the increased expression of YAP and TAZ had an effect on tumor cell proliferation, as determined by minichromosome maintenance 7, DNA replication licensing factor 7 expression. Their prognostic value was also assessed. In total, 127 patients who underwent radical surgery and were subjected to adjuvant radiation therapy due to squamous cell OCC were enrolled in the present study. The results demonstrated an evident effect as YAP expression increased in cancer-associated fibroblasts, which induced unfavorable prognosis in patients. In addition, a positive association between proliferation in cancer cells and YAP expression in stromal cells was observed. A lack of YAP expression in the cytoplasm of tumor cells was a factor for poor prognosis with regard to disease-free survival and disease specific survival. No statistically significant
\end{abstract}

Correspondence to: Dr Jolanta Szelachowska, Department of Oncology, Gynaecological Oncology Clinic, Wroclaw Medical University, Plac Hirszfelda 12, 53-413 Wroclaw, Poland

E-mail: jolanta.szelachowska@umed.wroc.pl

Key words: Yes-associated protein 1, TAZ, PTPN14, MCM7, head and neck cancer biology, head and neck cancer prognosis correlations between YAP and TAZ expression and PTPN14 expression were identified, nor was a correlation between cell proliferation and the presence of YAP and TAZ in tumor cells observed. The results indicated that YAP expression levels may support the development of personalized therapies for patients.

\section{Introduction}

The first-line therapy for carcinoma of the oral cavity floor and/or oral surface of the tongue (OCC) is surgery, which is typically combined with adjuvant radiation therapy when the disease is highly advanced. The basic prognostic factors that aid in the selection of patients for postoperative treatment are the status of the surgical margins and disease advancement. Additional factors that require the initiation of adjuvant therapy are the unfavorable prognostic factors observed upon histopathological examination such as close surgical margins, perineural invasion, lymphovascular invasion and extensive lymph node invasion (1). On the other hand, the histological grade of the tumor $(\mathrm{G})$ is controversial in regard to its prognostic significance in OCC $(2,3)$.

Unfortunately, a large proportion of patients develop locoregional recurrence $(4,5)$, which suggests that despite the early stage detection of the disease, a more aggressive treatment is required in some cases. Therefore, it is necessary to identify novel prognostic factors that may identify groups of patients with a high risk of recurrence. The Hippo pathway effectors YAP1/Yes-associated protein 1 (YAP) and transcriptional co-activator with PDZ-binding motif, WWTR1 (TAZ), potentially meet the criteria for oncogenes, and appear to be promising markers.

YAP and TAZ are close paralogues that are mainly involved in the transduction of signals in the Hippo signaling pathway (6). These proteins are transcriptional modules of the Hippo signaling pathway. They function as transcriptional coactivators that shift from the cytoplasm to the cell nucleus 
where, mainly via interactions with TEAD transcriptional factors, they induce the expression of genes responsible for proliferation, cell growth, epithelial-mesenchymal transition (EMT) and apoptosis inhibition (7).

From the classical point of view, YAP and TAZ meet the criteria for oncogenes. Amplification in the region of the 11q21-22 chromosome that encodes YAP is observed in many types of cancers, and in squamous cell carcinoma (SCC) of the oral cavity in $5-15 \%$ of cases (8). In addition, it was previously reported that YAP and TAZ are the nuclear transducers of mechanical signals produced by the stiffness of the extracellular matrix (ECM) and cell shape (9). This regulation requires Rho GTPase activity and actomyosin cytoskeleton tension, and is independent from the Hippo signaling pathway. YAP and TAZ activity is positively correlated with ECM stiffness $(9,10)$. Experimental studies have shown that in dense cell populations, where the contact between cells is maintained, YAP and TAZ are not active and they are located in the cytoplasm, whereas in sparse cell populations YAP and TAZ are located in the cell nucleus and trigger proliferation $(9,11)$. However, mechanical stretching of the cells may result in prolonged activation of RhoA and myosin, which in turn induces YAP/TAZ migration to the cell nucleus, stimulating proliferation even in contact-inhibited epithelial cells. YAP/TAZ may have a key role in the contact-induced inhibition of cell proliferation, as its dysregulation is one of the main markers of cancerous cell transformation (12). Nevertheless, it was observed that YAP, depending on the cell context, functions not only as a protooncogene but also as a carcinogenesis suppressor (13).

A few reports have been published recently highlighting the role of tyrosine-protein phosphatase non-receptor type 14 (PTPN14) as a suppressor of YAP, the key oncoprotein of the Hippo signaling pathway that controls organ development through the regulation of proliferation and cell apoptosis $(14,15)$. PTPN14 protein belongs to the family of protein tyrosine phosphatases, which is a large family of enzymes that are involved in the phosphorylation of tyrosine proteins and act contrarily to protein tyrosine kinases, being the key regulator of many physiological processes within a cell, such as metabolism, growth and cell differentiation (16). PTPN14 is a $130 \mathrm{kDa}$ non-receptor protein encoded by a gene located on 1q32.2 chromosome (17). In vitro studies have demonstrated that the dysregulated expression of this protein results in changes in the adhesion, growth and structure of the cell cytoskeleton (18). Depending on its localization within the cell, it regulates the maintenance of cell junctions and adhesion, as well as proliferation (19). Changes in PTNP14 levels during embryogenesis have also been revealed to influence transforming growth factor (TGF)- $\beta$ induction and EMT, inducing morphological and functional changes, and the acquisition of migratory features by the cells (20).

The minichromosome maintenance 7 , DNA replication licensing factor 7 (MCM7) protein is a marker of cell proliferation considered necessary for the initiation and continuation of DNA replication process in eukaryotic cells $(21,22)$. It has also been revealed that YAP/TAZ contributes to the proliferation of non-small cell lung cancer, and breast and head and neck cancers via the upregulation of MCM7 (23).

In our previous study conducted on a similar though slightly larger group of patients (24), it was demonstrated that there was a significant effect of MCM7 and PTPN14 proteins on the prognosis of patients with OCC. To the best of our knowledge, this was the first study that evaluated the expression of PTPN14 in human tumors. It was demonstrated that the high expression of PTPN14 was an unfavorable prognostic factor and its expression in $>75 \%$ of cancer cells allowed for the identification of the group patients with a high risk of relapse (24). These results inspired new experiments and the design of the present study in order to evaluate the potential relationship of the previously tested PTPN14 with YAP and TAZ.

The aim of the present study was to analyze the correlation between the expression of YAP and TAZ, and PTPN14 and to determine whether the increased expression of the YAP and TAZ proteins had an effect on the level of tumor cell proliferation as assessed by MCM7 levels. Their prognostic value in the group of patients recruited was also determined.

\section{Materials and methods}

Patients. Ethical approval from the Bioethical Commission at the Medical University of Wroclaw was obtained (no. KB299/2013); due to the retrospective nature of the study the need for written informed consent from patients was waived. In total, 127 patients ( 25 women and 102 men) treated by postoperative radiation at the Lower Silesian Oncology Center between 2000 and 2011 were enrolled in the present study. Enrollment criteria included the diagnosis of SCC of the oral cavity floor $(\mathrm{C} 04)$ or oral surface of the tongue $(\mathrm{CO2})$ and radical resection of the primary tumor (R0) with simultaneous surgery of the regional lymphatic system.

Disease advancement stage was defined in line with the 7th edition of TNM classification of 2010 (25). Table I presents the studied clinicopathological characteristics of the patients. All patients were subjected to postoperative radiation therapy and the irradiated area was the resection site with a margin and regional draining lymph node basin. The radiation dose was between 50 and $68 \mathrm{~Gy}$ (mean: $60 \mathrm{~Gy}$, median: $60 \mathrm{~Gy}$ ). The median time that lapsed between surgery to the start of radiation therapy was 74 days (mean: 77 days). The treatment that had been applied was in line with currently accepted standards of care (26).

During a 5-year follow-up, 37 patients (29\%) developed recurrence, of whom 8 patients $(6 \%)$ developed metastases without locoregional recurrence. The average time between surgery and the detection of recurrence was 12.8 months (4.6-39.4 months). The average time between surgery and death due to cancer progression was 19.3 months (8-60 months).

Immunohistochemical examination. Studies were performed on the tissue materials acquired during surgical treatment fixed in $10 \%$ buffered formalin, and then embedded in paraffin blocks. Immunohistochemical determination of YAP1 (clone H9; cat. no. sc-271134; Santa Cruz Biotechnology, Inc.; dilution: 1:100), TAZ (clone 1H9; cat. no. LS-C173295; LifeSpan Biosciences Seattle, WA, USA; dilution 1:150), PTPN14 (clone 448701; cat. no. MAB 4458; R\&D Systems, Inc.; dilution 1:200), MCM7: clone immunoglobulin (Ig)-G1 DCS-141.1 (cat. no. NCL-MCM7; Novocastra; dilution 1:30), $\alpha$-smooth muscle actin (SMA; clone 1A4; cat. no. IR611; Dako; Agilent Technologies, Inc.; ready-to-use), Podoplanin (clone D2-40; cat. 
Table I. Clinical characteristics of the patients recruited to the present study.

$\begin{array}{ll}\text { Characteristics } & \text { Number of patients }\end{array}$

Percentage $(\%)$

Sex

Male

Female

Primary site of the tumor in the oral cavity

Oral surface of the tongue

Both the floor and the tongue are affected-primary location

cannot be established

Histological grade of the tumor

G1

G2

G3

Keratosis features present

Keratodes

Akeratodes

$\mathrm{T}$ value

T1

T2

T4

$\mathrm{N}$ value

NO

N1

N3

TNM stage

II

III

IV

Scope of surgery

Partial resection of the tongue, oral cavity floor and mandible

Partial resection of the tongue and oral cavity floor

Partial resection of the tongue or oral cavity floor

Bilateral vs unilateral lymphadenectomy

\section{Bilateral}

Unilateral

Type of lymphadenectomy on the tumor side

Radical, using Crile's method

Functional

Selective

Type of lymphadenectomy on the opposite side of the tumor

None

Radiation therapy 2D vs. 3D

no. IR072; Dako; Agilent Technologies, Inc.; ready-to-use) and Vimentin (clone V9; cat. no. IR630; Dako; Agilent Technologies, Inc.; ready-to-use) was performed on $4 \mu \mathrm{m}$-thick paraffin sections mounted on salinized slides (S3003; cat. no. Dako; Agilent
Technologies, Inc.), which were then subjected to deparaffinization, rehydration and heat induced epitope retrieval performed using PT Link, with EnVision FLEX Target Retrieval Solution High $\mathrm{pH}$ used for $20 \mathrm{~min}$ incubation at $97^{\circ} \mathrm{C}$. An Autostainer 
Link48 was used to perform immunological testing using detection reagents EnVision FLEX (cat. no. K8002; Dako; Agilent Technologies, Inc.).

For each of the antibodies used, positive and negative controls were employed. The assessment of all of the specimens was conducted by two of the authors who used a light microscope (OLYMPUS BX41). For each protein, all visual fields were analyzed; the entire tissue specimen irrespective of its size was assessed, taking into account the percentage of positively stained cells and the intensity of staining.

A semi-quantitative method was used to evaluate MCM7, PTPN14, YAP and TAZ expression in SCC. The two immunohistochemical reaction parameters used were the percentage of cells with a positive cytoplasmic or nuclear reaction (the percentage of reactive tissue) and the intensity of reaction. The semi-quantitative ImmunoReactive Score (IRS) scale of Remmele and Stenger (27) was used to calculate the final immunohistochemical reaction results. In this approach, points were given depending on the percentage of reactive cells $(0-4$ points: $0,0 \% ; 1,1-25 \% ; 2,26-50 \% ; 3,51-75 \%$; and 4 , $>75 \%$ ) and the intensity of reaction ( $0-3$ points). The scores for these two parameters were multiplied to give the final result, which was referred to as the IRS factor or score (0-12 points).

The expression of YAP protein in the stromal compartment (cancer-associated fibroblasts; CAFs) of the tumor was calculated semi-quantitatively as negative (0: no staining), weak (1: either diffuse weak staining or strong staining in $<10 \%$ of the analyzed cells), intermediate (2: either diffuse intermediate staining or strong staining in $\geq 10-30 \%$ of the analyzed cells) and strong (3: defined as strong staining in $>30 \%$ of the analyzed cells). CAFs were confirmed by the positive immunohistochemical reactions for $\alpha$-SMA, vimentin and podoplanin (D2-40), which are all characteristic markers of CAFs (Fig. 1).

Statistical analysis. All statistical analyses were performed using the Statistica 10 (StatSoft, Inc.) package, employing the Kaplan-Meier method, Cox's proportional hazard model and Spearman's rank correlation coefficient. Evaluation of the prognostic value of the variables in the present study was performed using overall survival (OS), defined as the survival duration from the day of surgery to death, disease specific survival (DSS), defined as the survival duration from the day of surgery to death due to cancer progression, disease free survival (DFS), defined as the survival from the day of surgery to the recurrence of cancer (metastases and/or local locoregional relapse), and locoregional recurrence-free survival (LRFS) defined as the survival from the day of surgery until the onset of locoregional relapse.

\section{Results}

Expression patterns of the analyzed proteins. The expression of YAP was observed in two cell populations: Cancer cells (only cytoplasmic localization) and in the stromal compartment of the tumor (CAFs with nuclear-cytoplasmic distribution). The median IRS for YAP expression in cancer cells was 6 (range: 0-12, mean: 5.25 \pm 3.7 ; Fig. 2). In 23 patients, no YAP expression was observed in tumor cells. YAP expression in CAFs was identified in 82 patients; in 30 patients it was scored 1, in 28 patients scored 2 and in 24 patients scored 3 points on a 4-point scale (0-3; Fig. 2).
TAZ protein exhibited exclusively nuclear expression in cancer cells, without any immunoreactivity in the stroma (Fig. 2). The median IRS for TAZ expression was 6 (range: $0-12$; mean: $5 \pm 3.9$ ). In 33 patients no expression of this marker was observed in tumor cells. MCM7 expression tended to be exclusively nuclear in nature; it was observed in all 127 patients. The IRS median for MCM7 expression was 4 (range: 1-12; mean: $5 \pm 3.4$ ).

PTPN14 expression exhibited cytoplasmic localization. In the majority of cases it was weakly expressed. The median IRS for PTPN14 expression was 1 (range: $0-8$, mean: $2 \pm 1.99$ ). In 31 patients, no PTPN14 expression was observed in tumor cells.

Correlations between analyzed proteins and clinicopathological parameters. In the studied group of patients, a weak positive correlation between the percentage of cells showing MCM7 expression and the presence of YAP protein in the tumor stroma were observed $\left(r_{s}=0.207 ; \mathrm{P}=0.019\right)$. However, no significant correlation with TAZ or YAP expression was identified in tumor cells. In addition, a weak correlation between MCM7 and the percentage of cells containing PTPN14 protein was identified $\left(r_{s}=0.202 ; P=0.022\right)$. A weak negative correlation between YAP and TAZ expression in tumor cells was also noted $\left(r_{\mathrm{s}}=-0.220, \mathrm{P}=0.012\right)$.

A weak positive correlation was revealed between the histological grade of the tumor $\mathrm{G}$ and stromal expression of YAP $\left(r_{s}=0.275 ; \mathrm{P}=0.001\right)$ and the percentage of cells highlighted the expression of MCM7 as a proliferation marker $\left(\mathrm{r}_{\mathrm{s}}=0.175 ; \mathrm{P}=0.048\right)$.

Furthermore, a weak negative correlation between the size of the primary tumor (pT) and the percentage of cells exhibiting YAP expression $\left(\mathrm{r}_{\mathrm{s}}=-0.196 ; \mathrm{P}=0.028\right)$, and a weak positive correlation with the intensity of the color reaction PTPN14 $\left(\mathrm{r}_{\mathrm{s}}=0.184 ; \mathrm{P}=0.039\right)$, were observed. The present study also demonstrated a weak negative correlation between pTNM and the percentage of cells exhibiting YAP expression $\left(r_{\mathrm{s}}=-0.226 ; \mathrm{P}=0.010\right)$.

The results did not present a correlation between the level of PTPN14 expression, and YAP and TAZ proteins.

Impact of clinical features on patients' survival. Among the clinical and pathological factors under assessment only the disease advancement stage significantly affected the prognosis of patients following univariate analysis. Statistically significant differences in the 5-year survival rates of patients were revealed dependent on $\mathrm{pT}, \mathrm{pN}$ and $\mathrm{pTNM}$. The best prognosis was noted for patients with TNM stage II for whom the 5-year LRFS, DFS, DSS and OS were 92, 89, 89 and 66\%, respectively, whilst in patients with TNM stage III, the survival decreased to $78,73,73$ and $54 \%$, respectively, and to $60,50,52$ and $42 \%$, respectively for stage IV.

Impact of the analyzed protein expression on patients' survival. It was revealed that patients without YAP expression in the tumor cell cytoplasm had a significantly shorter DFS and DSS (Table II; Fig. 3A and B). No correlation between YAP protein and LRFS and OS was identified. YAP expression in the tumor stroma markedly influenced DFS and OS (Table II; Fig. 3D) while the TAZ expression level did not affect patient survival (Table II; Fig. 3C). 

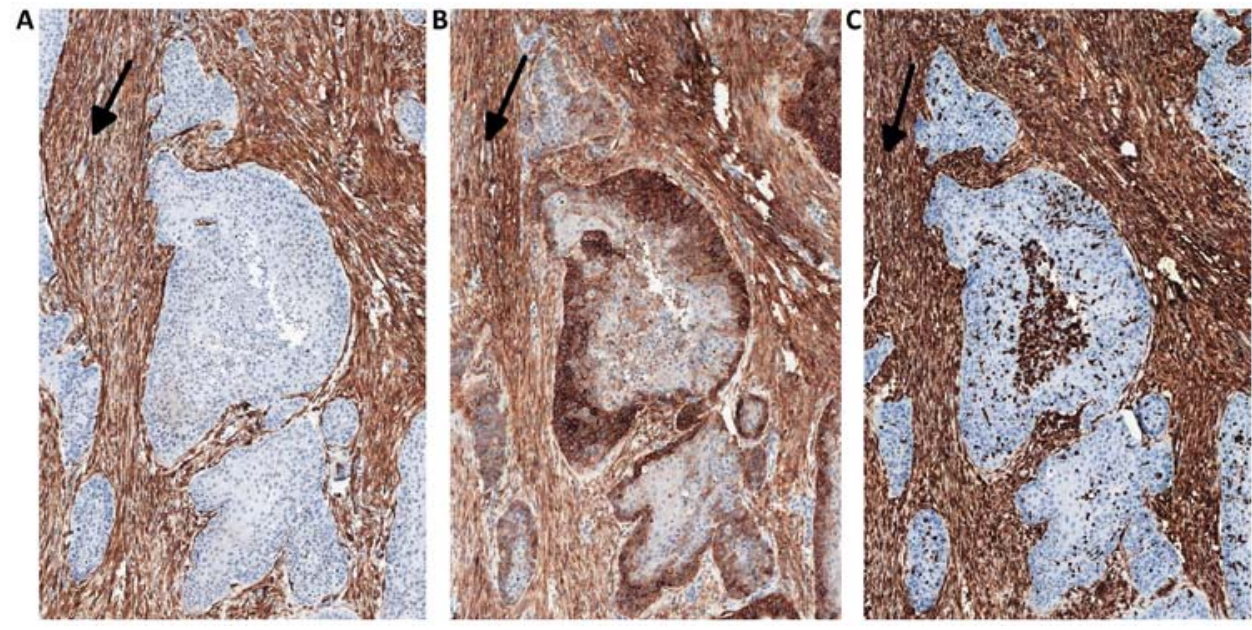

Figure 1. Serial sections immunostained for (A) $\alpha$-SMA, (B) podoplanin (D2-40) and (C) vimentin in CAFs (arrows). Magnification, x50. $\alpha$-SMA, $\alpha$-smooth muscle actin; $\mathrm{CAFs}$, cancer associated fibroblasts.
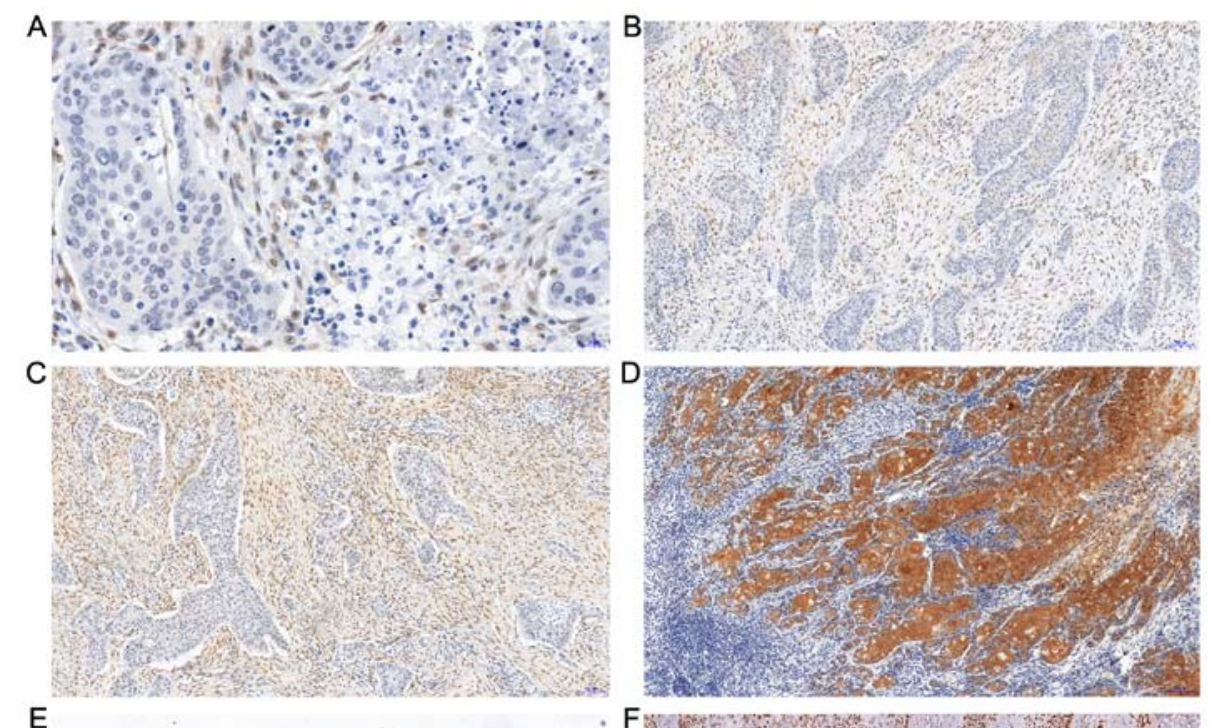

E

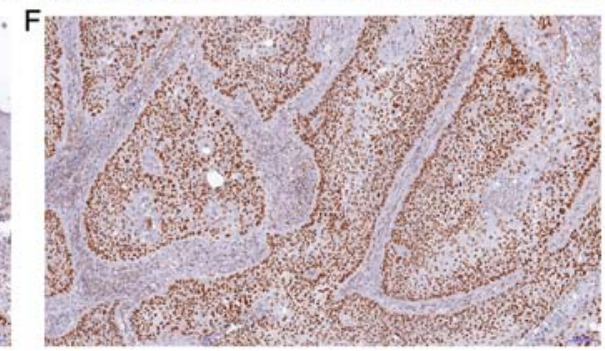

Figure 2. Immunohistochemical expression of YAP and TAZ proteins in SCC of the oral cavity floor or of the oral surface of the tongue. (A) No YAP immunoreactivity in cancer cells with moderate expression with nuclear localization in stromal compartment of the tumor (magnification, $\mathrm{x} 400$ ). (B and C) Enhanced reactivity of YAP in the stromal compartment of the tumor with nuclear distribution (magnification, x100). (D) Strong expression of YAP protein in squamous cancer cells with no reactivity in stromal compartment (magnification, x100; hematoxylin). (E) Strong nuclear expression of TAZ protein in dysplastic and cancer cells (magnification, x100). (F) Enhanced nuclear reactivity of TAZ in squamous cancer cells (magnification, x100). YAP, YAP1/Yes-associated protein 1; TAZ, transcriptional coactivator with PDZ-binding motif, WWTR1; SCC, squamous cell carcinoma.

Furthermore, similar to our previous report (24), patients with high expression levels of MCM7 in tumor cells had a significantly shorter LRFS, DFS, DSS and OS. In a group of patients with $>75 \%$ tumor cells exhibiting PTPN14 expression, the 5-year LRFS and DFS were significantly worse.

Multivariate analysis. The present multivariate analysis included the parameters of YAP and TAZ expression that had a statistically significant effect on survival or produced non-significant but marked prognostic trends in univariate analysis. In the multivariate analysis, patients' age had a significantly unfavorable influence on OS, and its effect on DFS and DSS was marked but not significantly different. High YAP expression in stromal cells at the level of 2 or 3 had an unfavorable effect on DFS and OS. No YAP expression in the cytoplasm of cancer cells was an independent unfavorable prognostic 
Table II. Percentage of 5-year LRFS, DFS, DSS and OS in the patients depending on the expression of MCM7, PTPN14, YAP, stromal YAP and TAZ in squamous carcinoma cells.

\begin{tabular}{|c|c|c|c|c|}
\hline & 5-year LRFS (\%) & 5-year DFS (\%) & 5-year DSS (\%) & 5-year OS (\%) \\
\hline \multicolumn{5}{|l|}{ МCM7\% } \\
\hline$\leq 25 \%$ & 88 & 85 & 85 & 64 \\
\hline$>25 \%$ & 71 & 64 & 65 & 49 \\
\hline P-value & 0.029 & 0.014 & 0.018 & 0.072 \\
\hline \multicolumn{5}{|c|}{ MCM7 INT } \\
\hline 1 & 86 & 81 & 83 & 61 \\
\hline 2 & 77 & 69 & 69 & 57 \\
\hline 3 & 59 & 54 & 54 & 37 \\
\hline Pvalue & 0.005 & 0.005 & 0.006 & 0.041 \\
\hline \multicolumn{5}{|c|}{ MCM7 IRS } \\
\hline$<4$ & 86 & 81 & 83 & 62 \\
\hline$\geq 4$ & 69 & 62 & 62 & 47 \\
\hline P-value & 0.018 & 0.009 & 0.005 & 0.054 \\
\hline \multicolumn{5}{|l|}{ YAP\% } \\
\hline$>0 \%$ & 79 & 75 & 76 & 39 \\
\hline $0 \%$ & 58 & 44 & 43 & 56 \\
\hline P-value & 0.12 & 0.011 & 0.007 & 0.11 \\
\hline \multicolumn{5}{|l|}{ YAPs } \\
\hline $0-1$ & 79 & 76 & 76 & 61 \\
\hline $2-3$ & 69 & 59 & 61 & 41 \\
\hline P-value & 0.17 & 0.060 & 0.11 & 0.064 \\
\hline \multicolumn{5}{|l|}{ TAZ\% } \\
\hline$>0 \%$ & 79 & 73 & 73 & 58 \\
\hline $0 \%$ & 62 & 57 & 59 & 39 \\
\hline P-value & 0.13 & 0.19 & 0.30 & 0.12 \\
\hline \multicolumn{5}{|c|}{ PTPN14 \% } \\
\hline$\leq 75 \%$ & 78 & 71 & 71 & 54 \\
\hline$>75 \%$ & 37 & 37 & 50 & 37 \\
\hline P-value & 0.007 & 0.033 & 0.13 & 0.24 \\
\hline
\end{tabular}

Significant results are highlighted in bold $(\mathrm{P}<0.05)$. LRFS, locoregional recurrence-free survival; DFS, disease-free survival; DSS, disease specific survival; OS, overall survival; MCM7, minichromosome maintenance 7, DNA replication licensing factor 7; PTPN14, tyrosine-protein phosphatase non-receptor type 14; YAP, YAP1/Yes-associated protein 1; TAZ, transcriptional co-activator with PDZ-binding motif, WWTR1.

factor for DFS and DSS (Table III), and was markedly different in the case of LRFS and OS. Multivariate analysis confirmed the findings of our previous paper (24): A high percentage $(>75 \%)$ of cells exhibiting cytoplasmic PTPN14 expression was an unfavorable prognostic factor for LRFS, DFS and DSS, and several times increased the risk of disease progression or death. In addition, $\mathrm{pT}, \mathrm{pN}$, and color reaction intensity with MCM7 protein were revealed to be independent prognostic factors for LRFS, DFS, DSS and OS (Table III).

\section{Discussion}

In the studied group, $\mathrm{pT}$ and $\mathrm{pN}$ were independent prognostic factors that significantly influenced patient survival. The results were in agreement with the previously established fact that the primary, important prognostic factors in patients with OCC are the disease advancement stage TNM, pT and pN $(25,28)$. In addition, the previously tested markers MCM7 and PTPN14 had a significant impact on the prognosis of patients. Of the newly tested proteins, YAP showed an effect on patient survival, while TAZ did not meet the criterion of a prognostic factor.

The hypothesis that MCM proteins may be good proliferation markers has been tested in many previous studies (29-32). One of the first reports on the prognostic significance of MCM2 as a cell proliferation marker in OCC was the work by our team (33) performed on a different, smaller group of patients. In this study the expression of MCM2 was compared with the recognized proliferation marker $\mathrm{Ki}-67$ and the results revealed a strong correlation between the expression levels of these two markers in oral SCC and additionally it was demonstrated to be a better prognostic factor than $\mathrm{Ki}-67$. In the case of the MCM7 protein, only one study has been reported that assessed the effect 
A

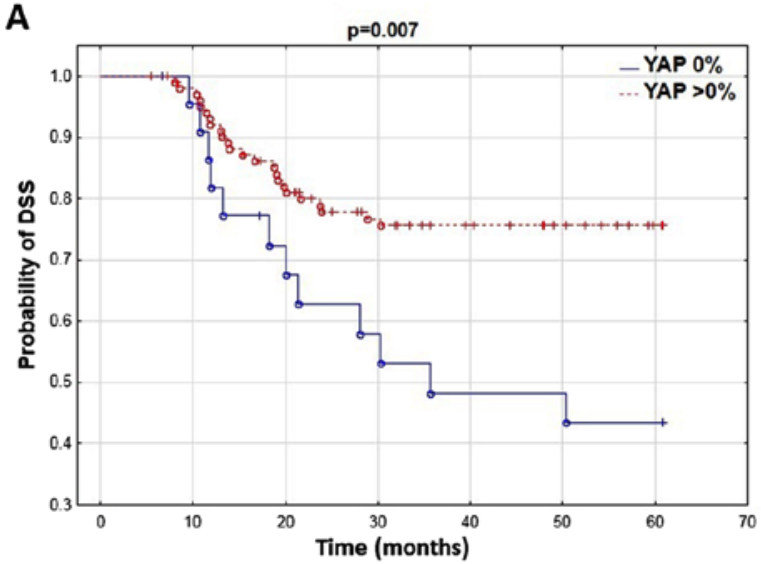

C

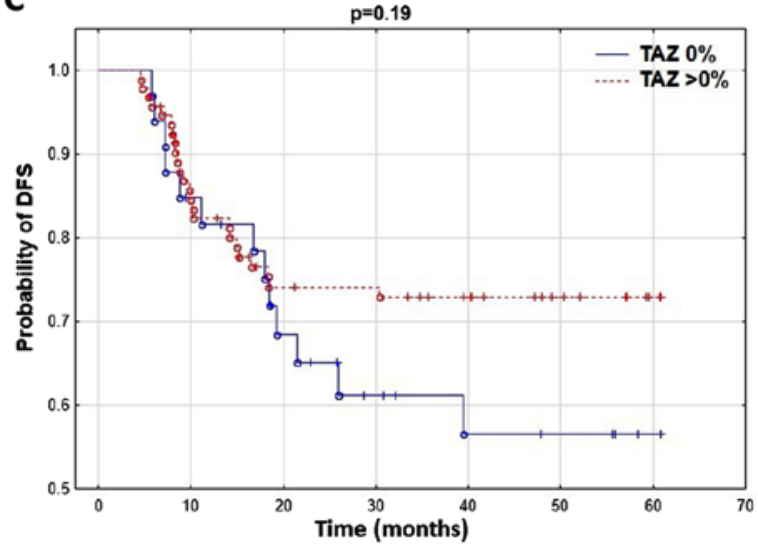

B

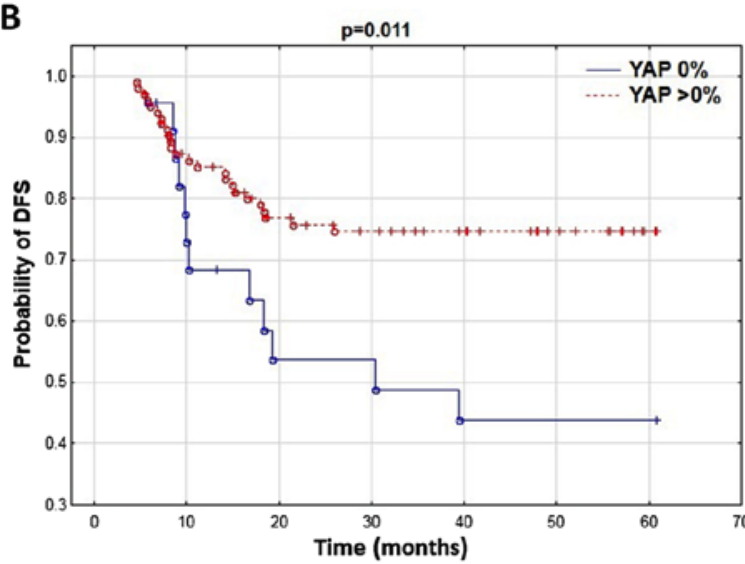

D

D $\quad 0.060$

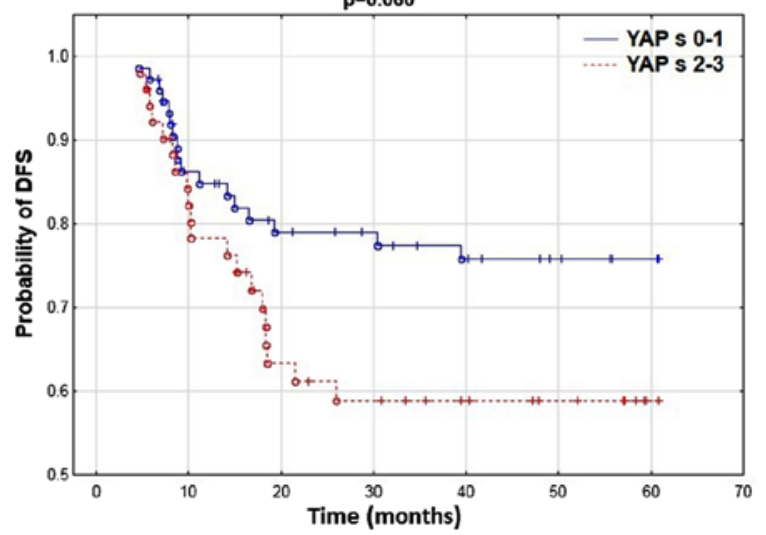

Figure 3. Comparison (using the Kaplan-Meier method) of (A) DSS curves depending on YAP expression in tumor cells, and DFS depending on (B) YAP and (C) TAZ expression in cancer cells and (D) YAPs in tumor stromal cells. DFS, disease-free survival; DSS, disease specific survival; YAP, YAP1/Yes-associated protein 1; TAZ, transcriptional coactivator with PDZ-binding motif, WWTR1.

of its expression on prognosis in OCC (30), in which shorter survival was typical for patients with MCM7 expression in $>49 \%$ of cancer cells. However, this effect was limited to a subgroup of TNM stage III and IV patients, and was not noticed in the group of stage I and II patients. On the other hand, in our present and previous studies, MCM7 expression had an effect on patient prognosis at all stages of advancement, and in the multivariate analysis, along with $\mathrm{pT}$ and $\mathrm{pN}$ grade, it was the only independent prognostic factor for each of the assessed survival parameters.

The relationship between proliferation and PTPN14 was described by Wadham et al (19), who noticed a correlation between PTPN14 translocation to the nucleus and proliferation initiation, which indicates its important role in the process. In the present study, PTPN14 was located in the cytoplasm and upon immunohistochemical examination, its nuclear expression was not observed. However, a high percentage of cells $(>75 \%)$ containing this protein were correlated with the studied proliferation marker MCM7 and was in turn related with poor prognosis.

Furthermore, in the present study high expression of PTPN14 had the highest effect on locoregional recurrence with a decreasing effect on DFS and DSS. Studies on Zebrafish (Danio reiro) embryos reported that PTPN14 overexpression induced TGF $\beta$ secretion and via the canonical Smad-related pathway induced changes in the expression of genes associated with EMT including Snail, Slug, ZEB1 and ZEB2, and the loss of E-cadherin expression, which resulted in morphological and functional changes and the acquisition of migratory features by the cells (20). It is possible that the high expression of PTPN14 results in the increased migration of cancer cells and, despite negative margins of resection, cancer cell deposits may be near the postoperative field, which is associated with an almost 6-fold higher risk of local recurrence.

In 2012 and 2013, three papers were published reporting that PTPN14 inhibits the activity of YAP by promoting its cytoplasmic location $(14,34,35)$. In these studies, it was noticed that the combination of PTPN14 protein with YAP inhibited its translocation to the nucleus and transcription of target genes for YAP. On the other hand, lack of PTPN14 resulted in YAP overexpression and increased cell migration (14,34). In 2013, another article was published indicating that PTPN14 was a negative regulator of YAP transcriptional activity. However, the inhibition of YAP translocation to the cell nucleus induced by PTPN14 was not described (15). In 2014, Wilson et al (36) demonstrated that PTPN14 not only directly, but also indirectly through activation of large tumor suppressor drosophila homolog 1, negatively regulated YAP and TAZ. However, the present study did not observe a correlation between PTPN14, and YAP and TAZ expression.

In the present study, TAZ was located only in the cancer cell nuclei and despite an evident tendency for a poorer prognosis in the group of patients whose cancer cells did not exhibit TAZ expression, no significant effect on survival was observed. In the case of YAP in cancer cells, only a cytoplasmic reaction was identified, while in CAFs a nuclear-cytoplasmic reaction was observed. In 
Table III. Final multivariate analysis for LRFS, DFS, OS and DSS.

\begin{tabular}{|c|c|c|c|c|}
\hline Prognostic factors & P-value & Hazard ratio (HR) & 95\% PU HR lower & 95\% PU HR upper \\
\hline \multicolumn{5}{|l|}{ LRFS } \\
\hline YAP $\%$ & 0.068 & 0.432 & 0.176 & 1.063 \\
\hline $\mathrm{pT}$ & 0.001 & 2.703 & 1.650 & 4.428 \\
\hline $\mathrm{pN}$ & 0.012 & 1.717 & 1.127 & 2.615 \\
\hline MCM7 INT & 0.001 & 3.091 & 1.729 & 5.527 \\
\hline PTPN14 \% & 0.001 & 6.077 & 2.069 & 17.854 \\
\hline \multicolumn{5}{|l|}{ DFS } \\
\hline YAP \% & 0.009 & 0.360 & 0.166 & 0.779 \\
\hline YAPs & 0.042 & 2.094 & 1.027 & 4.270 \\
\hline pT & 0.001 & 2.316 & 1.477 & 3.633 \\
\hline $\mathrm{pN}$ & 0.008 & 1.674 & 1.141 & 2.455 \\
\hline MCM7 INT & 0.001 & 2.700 & 1.608 & 4.535 \\
\hline PTPN14 \% & 0.006 & 4.262 & 1.507 & 12.054 \\
\hline Age & 0.061 & 1.041 & 0.998 & 1.087 \\
\hline \multicolumn{5}{|l|}{ DSS } \\
\hline YAP $\%$ & 0.004 & 0.327 & 0.153 & 0.703 \\
\hline pT & 0.001 & 2.355 & 1.522 & 3.645 \\
\hline $\mathrm{pN}$ & 0.001 & 1.932 & 1.314 & 2.840 \\
\hline MCM 7 INT & 0.001 & 2.436 & 1.478 & 4.015 \\
\hline PTPN14 \% & 0.016 & 3.891 & 1.283 & 11.805 \\
\hline Age & 0.061 & 1.044 & 0.998 & 1.093 \\
\hline \multicolumn{5}{|l|}{ OS } \\
\hline YAP $\%$ & 0.084 & 0.569 & 0.301 & 1.078 \\
\hline $\mathrm{pT}$ & 0.001 & 1.819 & 1.295 & 2.555 \\
\hline $\mathrm{pN}$ & 0.020 & 1.426 & 1.058 & 1.920 \\
\hline MCM 7 INT & 0.008 & 1.619 & 1.136 & 2.307 \\
\hline Age & 0.014 & 1.045 & 1.009 & 1.082 \\
\hline YAPs & 0.041 & 1.770 & 1.025 & 3.058 \\
\hline
\end{tabular}

Significant results are highlighted in bold $(\mathrm{P}<0.05)$. HR of recurrence or death due to carcinoma of the oral surface of the tongue or oral cavity floor depending on independent prognostic factors: YAP expression (percentage of cells with YAP: 0 vs. $>0 \%$ ), YAP expression in stromal cells (intensity 0-1 vs. 2-3), MCM7 expression (reaction intensity 1 vs. 2 vs. 3), PTPN14 expression (percentage of cells with PTPN14: 0-75 vs. $>75 \%$ ), size of primary tumor pT ( 1 vs. 2 vs. 3 vs. 4 ), regional lymph node involvement pN (0 vs. 1 vs. 2$)$, and age in years at diagnosis. LRFS, locoregional recurrence-free survival; DFS, disease-free survival; DSS, disease specific survival; OS, overall survival; HR, hazard ratio; PTPN14, tyrosine-protein phosphatase non-receptor type 14; YAP, YAP1/Yes-associated protein 1.

the present study, no cytoplasmic YAP expression in cancer cells was related with poor prognosis, and was an independent prognostic factor for DFS and DSS. Similar results highlighted YAP as a suppressor in SCC of the head and neck area as presented by Ehsanian et al (37), in 2010. This study demonstrated that cells with YAP knockdown exhibited increased proliferation, survival, migration abilities and resistance to cisplatin. On the other hand, YAP expression increased cell apoptosis and chemosensitivity; however, YAP's function as a suppressor may be dysregulated by AKT or $\Delta \mathrm{Np} 63$ (37). Nevertheless, the majority of papers indicate that there is a correlation between YAP and TAZ overexpression in cancer cells and the presence of aggressive clinicopathological features and unfavorable prognosis of cancer patients and chemoresistance (38-41).

At the same time some findings have suggested that YAP may function not only as a protooncogene but also as a suppressor of tumors depending on the cell context $(13,42)$. Recently, papers have been published that report that YAP and TAZ may suppress WNT signal transduction amongst other signals by sequestering $\beta$-catenin in the cytoplasm or intensifying its degradation $(43,44)$.

It was previously revealed that YAP is silenced in highly aggressive human colorectal cancers and its re-expression may limit cancer growth, which indicates its potential role as a suppressor $(13,45)$. It seems that YAP and TAZ expression in the cytoplasm function as $\beta$-catenin inhibitors, whereas with the nuclear localization they are positive mediators of WNT-associated transformation (44).

Furthermore, it was noted that in normal hematologic cells oncogene-mediated DNA damage induces ataxia telangiectasia mutated and then JUN N-terminal kinase activation, which phosphorylates 14-3-3 adaptor protein causing Abelson tyrosine-protein kinase 1 (ABL1) to shift from the cytoplasm to the cell nucleus $(46,42)$. Upon entry into the cell nucleus, ABL1 phosphorylates the YAP tyrosine residue. 
Phosphorylated YAP forms a complex with the TP73 tumor suppressor and maintains the transcription of pro-apoptotic genes such as $B A X$ and PUMA (42). MST1-induced activation of the Hippo signaling pathway, via phosphorylation of serine residues YAP, inhibits its pro-apoptotic activity (42). Low expression of YAP prevents the apoptosis induced by ABL1 in the case of DNA damage in hematologic cancers. On the other hand, the re-occurrence of YAP expression in tumor cells promotes apoptosis and growth arrest (42).

A possible explanation for this contradictory information concerning YAP function in cancer is that it may form complexes with various transcriptional factors that have different functions depending on the cancer type. TAZ and YAP may have varying effects on carcinogenesis by associating with different transcriptional factors depending on the cell context.

The present study observed a weak positive correlation between YAP expression in CAFs and the percentage of tumor cells presenting MCM7 expression. Furthermore, the results demonstrated an unfavorable effect of high YAP expression in CAFs on DFS and OS. This finding suggests a direct relationship between the presence of YAP in stromal cells and the increased proliferation of cancer cells and poorer patient prognoses. This phenomenon may be explained by earlier studies that suggested that YAP activation in CAFs promoted stiffening of the matrix by increased deposition of collagen (47). Furthermore, stiffening of the matrix causes tension within the CAFs, which results in Src kinase activation and YAP translocation to the nucleus and as a result, YAP and TAZ promote the expression of regulators of the cytoskeleton such as anillin actin binding protein and diaphanous related formin 3 , and stabilizing actomyosin proteins. This further stiffens the matrix, increases proliferation and results in the formation of the self-enforcing loop during carcinogenesis (47).

In conclusion, the present demonstrated the significant effect of increased YAP expression in cancer-associated fibroblasts on the unfavorable prognosis of patients with squamous OCC. Additionally, a weak positive correlation was confirmed between proliferation in cancer cells and YAP expression in the stroma. This finding highlights the key role of the ECM in cancer progression. The results also demonstrated that the lack of YAP expression in the cytoplasm of tumor cells may be a poor prognostic factor for DFS and DSS, suggesting its suppressive effect on cancer in this region. No statistically significant correlations were identified between the level of YAP and TAZ expression and PTPN14, nor was a correlation between the level of cell proliferation and the presence of YAP and TAZ protein in tumor cells observed. However, due to the fact that the findings reported by other authors are inconclusive, the present observations require further validation in a future study on another group of patients.

\section{Acknowledgements}

Not applicable.

\section{Funding}

The presented results of studies performed as part of the subject as per records in the Simple system number ST.C280.17.010 and SUB.C280.19.050 were financed through a statutory subsidy by the Minister of Science and Higher Education.

\section{Availability of data and materials}

The datasets generated and/or analyzed during the current study are not publicly available due to our institutional policy but are available from the corresponding author on reasonable request.

\section{Authors' contributions}

JS undertook study design, data collection, analysis of data, manuscript preparation and the Literature search. PD performed data collection, analysis of data, manuscript preparation and microphotographs. KRW analyzed the data, prepared the manuscript and took microphotographs. AH analyzed the data and reviewed the manuscript. DZ and KL were involved in drafting the manuscript, revising it critically for important intellectual content and given final approval of the version to be published, in addition they took part in th eacquisition of data and analysis and interpretation of data. AM analyzed the data and reviewed the manuscript. ELW undertook the literature search, data collection and manuscript preparation. AP analyzed the data, prepared the manuscript and took microphotographs. RM was responsible for study design, analyzing the data and reviewing the manuscript.

\section{Ethics approval and consent to participate}

The consent of the bioethical commission was obtained (consent no. KB299/2013).

\section{Patient consent for publication}

Due to the retrospective nature of the work and the lack of influence of the conducted research on the treatment method, no written consent for conducting the research was obtained from patients.

\section{Competing interests}

The authors declare that they have no competing interests.

\section{References}

1. Protocol for the Examination of specimens from Patients with Carcinomas of the Lip and Oral Cavity. https://cap.objects.frb. io/protocols/cp-headandneck-lip-oralcavity-17protocol-4001.pdf Accessed May 1, 2017.

2. Lindenblatt Rde C, Martinez GL, Silva LE, Faria PS, Camisasca DR and Lourenço Sde Q: Oral squamous cell carcinoma grading systems-analysis of the best survival predictor. J Oral Pathol Med 41:34-39, 2012.

3. Bhargava A, Saigal S and Chalishazar M: Histopathological grading systems in oral squamous cell carcinoma: A review. J Int Oral Health 2: 1-9, 2010.

4. Brandwein-Gensler M and Smith RV: Prognostic indicators in head and neck oncology including the new 7 th edition of the AJCC staging system. Head Neck Pathol 4: 53-61, 2010.

5. Zwetyenga N, Majoufre-Lefebvre C, Siberchicot F, Demeaux H and Pinsolle J: Squamous-cell carcinoma of the tongue: Treatment results and prognosis. Rev Stomatol Chir Maxillofac 104: 10-17, 2003 (In French).

6. Pan D: The hippo signaling pathway in development and cancer. Dev Cell 19: 491-505, 2010.

7. Johnson R and Halder G: The two faces of Hippo: Targeting the Hippo pathway for regenerative medicine and cancer treatment. Nat Rev Drug Discov 13: 63-79, 2014. 
8. Baldwin C, Garnis C, Zhang L, Rosin MP and Lam WL: Multiple microalterations detected at high frequency in oral cancer. Cancer Res 65: 7561-7567, 2005.

9. Dupont S, Morsut L, Aragona M,EnzoE, Giulitti S, Cordenonsi M, Zanconato F, Le Digabel J, Forcato M, Bicciato S, et al: Role of YAP/TAZ in mechanotransduction. Nature 474: 179-183, 2011.

10. Sanz-Moreno V, Gaggioli C, Yeo M, Albrengues J, Wallberg F, Viros A, Hooper S, Mitter R, Féral CC, Cook M, et al: ROCK and JAK1 signaling cooperate to control actomyosin contractility in tumor cells and stroma. Cancer Cell 16: 229-245, 2011.

11. Aragona M, Panciera T, Manfrin A, Giulitti S, Michielin F, Elvassore N, Dupont S and Piccolo S: A mechanical checkpoint controls multicellular growth through YAP/TAZ regulation by actin-processing factors. Cell 154: 1047-1059, 2013.

12. Zhao B, Wei X, Li W, Udan R, Yang Q, Kim J, Xie J, Ikenoue T, $\mathrm{Yu}$ J, Li L, et al: Inactivation of YAP oncoprotein by the Hippo pathway is involved in cell contact inhibition and tissue growth control. Genes Dev 21: 2747-2761, 2007.

13. Barry ER, Morikawa T, Butler BL, Shrestha K, de la Rosa R, Yan KS, Fuchs CS, Magness ST, Smits R, Ogino S, et al: Restriction of intestinal stem cell expansion and the regenerative response by YAP. Nature 493: 106-110, 2013.

14. Liu X, Yang N, Figel SA, Wilson KE, Morrison CD, Gelman IH and Zhang J: PTPN14 interacts with and negatively regulates the oncogenic function of YAP. Oncogene 32: 1266-1273, 2013.

15. Huang JM, Nagatomo I, Suzuki E, Mizuno T, Kumagai T, Berezov A, Zhang H, Karlan B, Greene MI and Wang Q: YAP modifies cancer cell sensitivity to EGFR and survivin inhibitors and is negatively regulated by the non-receptor type protein tyrosine phosphatase 14. Oncogene 32: 2220-2229, 2013.

16. Neel BG and Tonks NK: Protein tyrosine phosphatases in signal transduction. Curr Opin Cell Biol 9: 193-204, 1997.

17. Gyapay G, Morissette J, Vignal A, Dib C, Fizames C, Millasseau P, Marc S, Bernardi G, Lathrop M and Weissenbach J: The 1993-94 Généthon human genetic linkage map. Nat Genet 7: 246-339, 1994.

18. Ogata M, Takada T,Mori Y,Uchida Y, Miki T, Okuyama A, Kosugi A, Sawada M, Oh-hora M and Hamaoka T: Regulation of phosphorylation level and distribution of PTP36, a putative protein tyrosine phosphatase, by cell-substrate adhesion. J Biol Chem 274: 20717-20724, 1999.

19. Wadham C, Gamble JR, Vadas MA and Khew-Goodall Y: Translocation of protein tyrosine phosphatase Pez/PTPD2/PTP36 to the nucleus is associated with induction of cell proliferation. J Cell Sci 113: 3117-3123, 2000.

20. Wyatt L, Wadham C, Crocker LA, Lardelli $M$ and Khew-Goodall Y: The protein tyrosine phosphatase Pez regulates TGFbeta, epithelial-mesenchymal transition, and organ development. J Cell Biol 178: 1223-1235, 2007.

21. Ishimi Y: A DNA helicase activity is associated with an MCM4 -6 , and -7 protein complex. J Biol Chem 272: 24508-24513, 1997

22. Labib K, Tercero JA and Diffley JF: Uninterrupted MCM2-7 function required for DNA replication fork progression. Science 288: 1643-1647, 2000.

23. Lo Sardo F, Forcato M, Sacconi A, Capaci V, Zanconato F, Di Agostino S, Del Sal G, Pandolfi PP, Strano S, Bicciato S and Blandino G: MCM7 and its hosted miR-25, 93 and 106b cluster elicit YAP/TAZ oncogenic activity in lung cancer. Carcinogenesis 38: 64-75, 2017.

24. Szelachowska J: Różnice w długości przeżycia chorych na płaskonabłonkowego raka dna jamy ustnej lub trzonu języka w zależności od poziomu ekspresji białka MCM7, RB, E-kadheryny, p16, TGFb i PTPN14 w komórkach guza. Wrocław: Uniwersytet Medyczny im. Piastów Śląskich 2015 (In Polish).

25. Edge S, Byrd D, Compton C, Fritz A, Greene F and Trotti A (eds): AJCC Cancer Staging Manual. 7th edition. Springer, New York, NY, 2010.

26. National Comprehensive Cancer Network. Bone Cancer (Version 1.2019). https://www.nccn.org/professionals/physician gls/pdf/head-and-neck.pdf. [Online] Accessed on 2019.05.07

27. Remmele W and Stegner HE: Recommendation for uniform definition of an immunoreactive score (IRS) for immunohistochemical estrogen receptor detection (ER-ICA) in breast cancer tissue. Pathologe 8: 138-140, 1987.

28. Leemans CR, Tiwari R, Nauta JJ, van der Waal I and Snow GB: Recurrence at the primary site in head and neck cancer and the significance of neck lymph node metastases as a prognostic factor. Cancer 73: 187-190, 1994.

29. Gueiros LA, Coletta RD, Kowalski LP and Lopes MA: Clinicopathological features and proliferation markers in tongue squamous cell carcinomas. Int J Oral Maxillofac Surg 40: 510-515, 2011.
30. Tamura T, Shomori K, Haruki T, Nosaka K, Hamamoto Y, Shiomi T, Ryoke K and Ito H: Minichromosome maintenance-7 and geminin are reliable prognostic markers in patients with oral squamous cell carcinoma: Immunohistochemical study. J Oral Pathol Med 39: 328-334, 2010.

31. Feng CJ, Li HJ, Li JN, Lu YJ and Liao GQ: Expression of Mcm7 and Cdc6 in oral squamous cell carcinoma and precancerous lesions. Anticancer Res 28: 3763-3769, 2008

32. Torres-Rendon A, Roy S, Craig GT and Speight PM: Expression of $\mathrm{Mcm} 2$, geminin and Ki67 in normal oral mucosa, oral epithelial dysplasias and their corresponding squamous-cell carcinomas. Br J Cancer 100: 1128-1134, 2009.

33. Szelachowska J, Dziegiel P, Jelen-Krzeszewska J, Jelen M, Matkowski R, Pomiecko A, Spytkowska B, Jagas M, Gisterek I and Kornafel J: Mcm-2 protein expression predicts prognosis better than Ki-67 antigen in oral cavity squamocellular carcinoma. Anticancer Res 26: 2473-2478, 2006.

34. Michaloglou C, Lehmann W, Martin T, Delaunay C, Hueber A, Barys L, Niu H, Billy E, Wartmann M, Ito M, et al: The tyrosine phosphatase PTPN14 is a negative regulator of YAP activity. PLoS One 8: e61916, 2013.

35. Wang W, Huang J, Wang X, Yuan J, Li X, Feng L, Park JI and Chen J: PTPN14 is required for the density-dependent control of YAP1. Genes Dev 26: 1959-1971, 2012.

36. Wilson KE, Li YW, Yang N, Shen H, Orillion AR and Zhang J: PTPN14 forms a complex with Kibra and LATS1 proteins and negatively regulates the YAP oncogenic function. J Biol Chem 289: 23693-23700, 2014.

37. Ehsanian R, Brown M, Lu H, Yang XP, Pattatheyil A, Yan B, Duggal P, Chuang R, Doondeea J, Feller S, et al: YAP dysregulation by phosphorylation or $\Delta \mathrm{Np} 63$-mediated gene repression promotes proliferation, survival and migration in head and neck cancer subsets. Oncogene 29: 6160-6171, 2010.

38. Ge L, Smail M, Meng W, Shyr Y, Ye F, Fan KH, Li X, Zhou HM and Bhowmick NA: Yes-associated protein expression in head and neck squamous cell carcinoma nodal metastasis. PLoS One 6: 27529, 2011.

39. Jerhammar F, Johansson AC, Ceder R, Welander J, Jansson A, Grafström RC, Söderkvist P and Roberg K: YAP1 is a potential biomarker for cetuximab resistance in head and neck cancer. Oral Oncol 50: 832-839, 2014.

40. Li Z, Wang Y, Zhu Y, Yuan C, Wang D, Zhang W, Qi B, Qiu J, Song X, Ye J, et al: The Hippo transducer TAZ promotes epithelial to mesenchymal transition and cancer stem cell maintenance in oral cancer. Mol Oncol 9: 1091-1105, 2015.

41. Zheng L, Xiang C, Li X, Guo Q, Gao L, Ni H, Xia Y and Xi T: STARD13-correlated ceRNA network-directed inhibition on YAP/TAZ activity suppresses stemness of breast cancer via co-regulating Hippo and Rho-GTPase/F-actin signaling. J Hematol Oncol 30: 72, 2018.

42. Cottini F, Hideshima T, Xu C, Sattler M, Dori M, Agnelli L, ten Hacken E, Bertilaccio MT, Antonini E, Neri A, et al: Rescue of Hippo coactivator YAP1 triggers DNA damage-induced apoptosis in hematological cancers. Nat Med 20: 599-606, 2014.

43. Attisano L and Wrana JL: Signal integration in TGF- $\beta$, WNT, and Hippo pathways. F1000Prime Rep 5: 17, 2013.

44. Azzolin L, Panciera T, Soligo S, Enzo E, Bicciato S, Dupont S, Bresolin S, Frasson C, Basso G, Guzzardo V, et al: YAP/TAZ incorporation in the $\beta$-catenin destruction complex orchestrates the Wnt response. Cell 158: 157-170, 2014.

45. Zanconato F, Cordenonsi M and Piccolo S: YAP/TAZ at the Roots of Cancer. Cancer Cell 13: 783-803, 2016.

46. Levy D, Adamovich Y, Reuven N and Shaul Y: Yap1 phosphorylation by c-Abl is a critical step in selective activation of proapoptotic genes in response to DNA damage. Mol Cell 29: 350-361, 2008.

47. Calvo F, Ege N, Grande-Garcia A, Hooper S, Jenkins RP, Chaudhry SI, Harrington K, Williamson P, Moeendarbary E, Charras G and Sahai E: Mechanotransduction and YAP-dependent matrix remodelling is required for the generation and maintenance of cancer-associated fibroblasts. Nat Cell Biol 15: 637-646, 2013.

This work is licensed under a Creative Commons Attribution-NonCommercial-NoDerivatives 4.0 International (CC BY-NC-ND 4.0) License. 